\section{Summary}

The intermediate lobe of the amphibian pituitary is poorly vascularized but receives direct aminergic and peptidergic innervations. In addition, the close contacts between the intermediate and neural lobes suggest that the latter may directly influence the activity of melanotropic cells. In this study, we have applied the indirect immunofluorescence technique to demonstrate the presence of three novel neuropeptides in the neurointermediate lobe complex of the frog pituitary. Fibers containing atrial natriuretic factor (ANF) and melanin-concentrating hormone $(\mathrm{MCH})$ have been visualized in the neural lobe, while numerous nerve terminals containing the neuropeptide tyrosine (NPY) have been observed among the parenchymal cells of the intermediate lobe. These morphological data support recent findings concerning a role for $\mathrm{ANF}, \mathrm{MCH}$ and NPY in the control of melanotropin secretion in amphibians.

\section{ADRESSES}

A.C. Andersen, J.M. Danger, P. Netchitailo, $H$. Vaudry : Groupe de recherche en endocrinologie moléculaire, UA CNRS 650, Unité alliée à l'Inserm, Faculté des sciences, Université de Rouen, 76130 Mont-Saint-Aignan, (France).

G. Pelletier : Groupe du CRM en endocrinologie moléculaire, Le centre hospitalier de l'université Laval, Québec, G1V 4G2, (Canada).

M. Cantin : Groupe du CRM en hypertension, Institut de recherches cliniques de Montréal, Québec, H2W 1R7, (Canada).

A.N. Eberlé : Département de recherche, Kanton hospital, 4031 Bâle, (Suisse).

\section{TIRÉS A PART}

H. Vaudry : UA Cnrs 650, Faculté des sciences, Université de Rouen, 76130 Mont-SaintAignan.

$m / s n^{\circ} 5$ ool. 3, mai 87

\title{
Localisation immunohistochimique de 3 neuropeptides (NPY, ANF, MCH) dans le complexe neurointermédiaire de l'hypophyse de grenouille
}

\author{
Ann C. Andersen, Jean-Michel Danger, \\ Pierre Netchitaillo, Georges Pelletier, \\ Marc Cantin, Alex N. Eberlé, \\ Hubert Vaudry
}

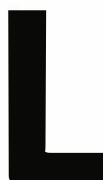

e lobe intermédiaire de l'hypophyse est une glande endocrine très faiblement vascularisée, voire totalement avasculaire, qui reçoit en revanche une innervation aminergique directe, composée, selon les espèces, de fibres libérant de la dopamine [1], de la norépinéphrine [2], de l'acide gamma-aminobutyrique [3] et (ou) de la sérotonine [4]. Chez les amphibiens, la pars intermedia possède en outre une innervation peptidergique. Par les techniques immunohistochimiques, on a pu identifier dans le lobe intermédiaire des fibres contenant la mésotocine [5], la corticolibérine [6] et la thyrolibérine [7]. Récemment, trois nouveaux neuropeptides ont été mis en évidence dans le système nerveux central des mammiferes : le neuropeptide tyrosine ou NPY [8], le facteur natriurétique auriculaire ou ANF [9] et le facteur d'agrégation des mélanophores ou MCH [10]. Des systèmes neuronaux à NPY, à $\mathrm{ANF}$ et à $\mathrm{MCH}$ ont été également identifiés dans le cerveau des amphibiens et des faisceaux de fibres contenant chacun de ces neuropeptides ont été observés dans l'éminence médiane et la tige pituitaire [11-13].

Dans le présent travail, nous montrons que, chez la grenouille verte Rana ridibunda, de nombreuses fibres à $\mathrm{ANF}$ et à $\mathrm{MCH}$ innervent le lobe nerveux de l'hypophyse tandis que les terminaisons à NPY sont particulièrement abondantes dans le lobe intermédiaire.

\section{Méthodes}

Préparation des tissus. Les grenouilles mâles adultes sont perfusées par voie intracardiaque avec $50 \mathrm{ml}$ de fixateur de McLean [14]. Les cerveaux sont disséqués en maintenant l'hypophyse en place, post-fixés pendant trois heures dans le même fixateur, puis rincés dans une solution de saccharose à $30 \%$ dans un tampon phosphate (PBS 0,1 M ; pH 7,4). Les cerveaux sont alors congelés et inclus dans un milieu d'enrobage (O.C.T. Tissu Teck). Des coupes parasagittales sériées de 8 $\mu \mathrm{m}$ d'épaisseur sont effectuées au cryotome (Reichert Jung).

Immunofluorescence. Les coupes de cerveau sont incubées pendant une nuit avec l'un des antisérums suivants : anti-NPY (1/400), antiANF $(1 / 200)$ ou anti-MCH $(1 / 200)$. Les dilutions des antisérums sont effectuées dans le tampon PBS contenant $1 \%$ d'albumine sérique humaine et $0,03 \%$ de Triton. Après rinçage, les coupes sont incubées avec une solution de $\boldsymbol{\gamma}$-globulines de mouton anti-IgG de lapin conjuguées à l'isothiocyanate de fluorescéine (Nordic Lab) diluée au 1/60e.

Après rinçage, les coupes sont contre-colorées au bleu Evans et 
Figure 1.

Locelisation per

lo technique

d'immuno-

fluorescence

indirecte du

NPY (o), do

I'ANF (b) ot du

MCH (c) dons

I'hypophyse. Les

photographies

représentent des

coupes

parasagittales de

l'hypophyse de

grenouille.
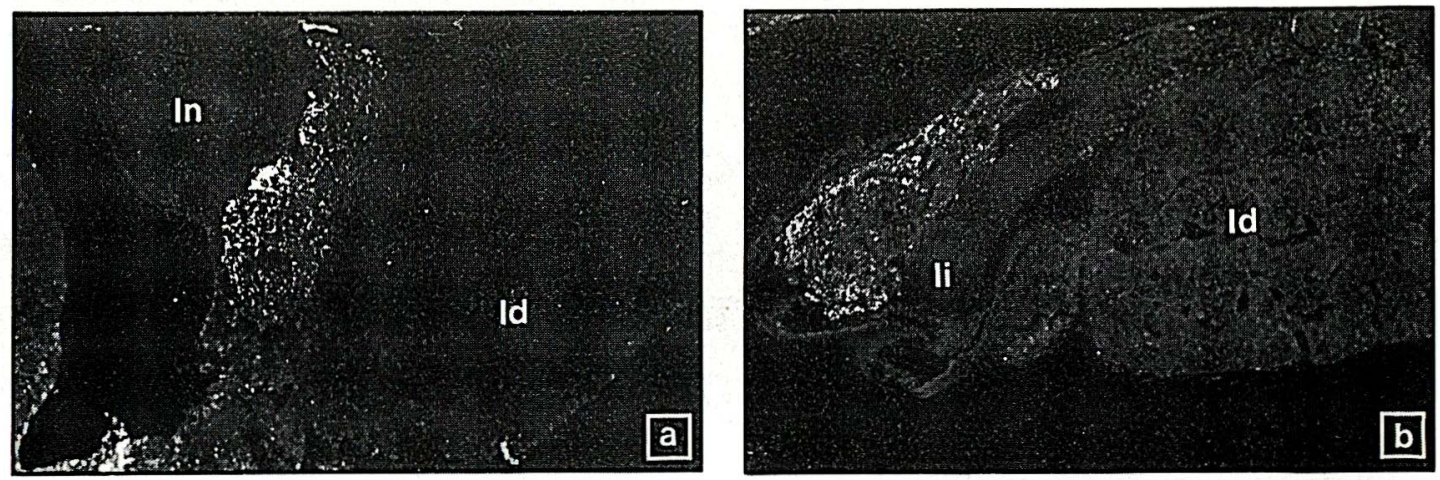

b montées dans du glycérol tamponné avec du $\operatorname{PBS}(1: 1)$. Les structures immunoréactives sont observées et photographiées en lumière UV (microscope Leitz Orthoplan). Les contrôles sont effectués en incubant les coupes avec des antisérums préabsorbés avec les peptides synthétiques homologues et hétérologues.

\section{Résultats et discussion}

Par la technique d'immunofluorescence indirecte, nous montrons l'existence d'un riche réseau de fibres contenant un peptide apparenté au NPY dans la pars intermedia de l'hypophyse de grenouille (figure 1a). Ces fibres sont réparties dans tout le parenchyme du lobe intermédiaire avec toutefois une concentration plus intense dans la région bordant le lobe nerveux. Quelques rares fibres à NPY sont aussi présentes dans le lobe nerveux au niveau de la frontière pars nervosa - pars intermedia.

Les fibres contenant l'ANF et le $\mathrm{MCH}$ sont principalement localisées dans le lobe nerveux de l'hypophyse (figures $1 b$, et $c$ ). L'ensemble du lobe nerveux contient une importante densité de terminaisons marquées par l'antisérum contre le $\mathrm{MCH}$ (figure 1c). La présence d'ANF dans la pars nervosa est plus discrète : la majo-

In : lobe nerveux; li : lobe intermédiaire; Id : lobe distal. $a: \times 110$ b: $\times 75$ c: $\times 70$.

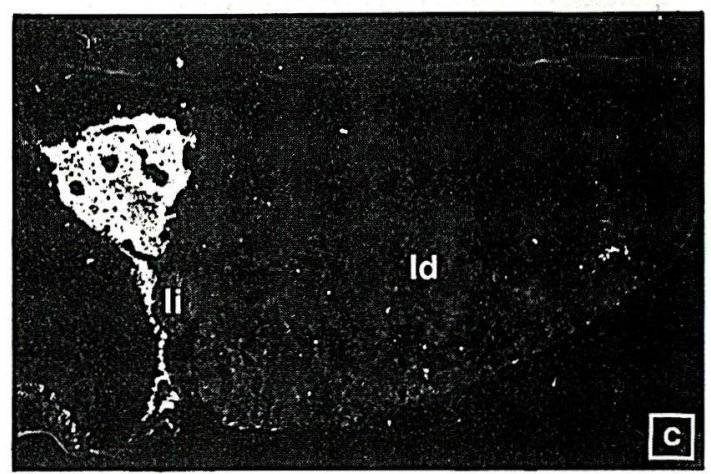

des fibres contenant l'ANF se trouve concentrée à la périphérie du lobe nerveux, principalemert dans la région bordant l'éminence médiane (figure 1b). Le lobe intermédiaire ne présente aucune structure marquée par l'anticorps anti-MCH, et seulement quelques rares fibres contenant l'ANF. Pour chacun des trois peptides étudiés, aucune immunoréactivité n'a pu être détectée dans le lobe distal de l'hypophyse.

Des coupes contrôles ont été effectuées pour déterminer la spécificité du marquage. Aucune réaction positive n'est observée lorsque chacun des anticorps est immunoabsorbé avec l'antigène homologue $\left(10^{-6} \mathrm{M}\right)$. Au contraire, le marquage n'est pas affaibli lorsque les anticorps sont incubés avec l'un des peptides hétérologues ou avec les peptides suivants : peptide pancréatique aviaire (APP), somatostatine, ou angiotensine II.

Les fibres du lobe nerveux contenant une immunoréactivité de type NPY, ANF ou MCF proviennent vraisemblablement des péricaryons que nous avons localisés dans l'hypothalamus anté- rieur et dans l'infundibulum [11-13]. Les corps cellulaires à $\mathrm{MCH}$ sont essentiellement situés dans le noyau préoptique et accessoirement dans le noyau infundibulaire dorsal $[13,15]$. Les fibres à $\mathrm{MCH}$ détectées dans le lobe nerveux proviennent donc exclusivement de neurones du diencéphale. Les péricaryons à NPY et ANF étant abondants dans l'aire préoptique et dans le noyau infundibulaire dorsal [11, 12], il est probable que les terminaisons du lobe nerveux contenant ces neuropeptides ont, elles aussi, une origine hypothalamique. Toutefois, des corps cellulaires à NPY ou ANF sont également observés dans de nombreuses aires du cerveau telles que le pallium (télencéphale), la couche grise superficielle du toit optique, le noyau interpédonculaire et le noyau du cervelet (mésencéphale) [11, 12]. De ce fait, on ne peut exclure que certaines fibres à NPY ou ANF du lobe nerveux n'aient une origine extrahypothalamique.

Compte tenu de la densité de l'innervation à NPY observée au niveau du lobe intermédiaire et de l'intense immunoréactivité de type 
ANF et $\mathrm{MCH}$ contenue dans le lobe nerveux, nos résultats suggèrent que ces trois neuropeptides jouent le rôle de neurohormones hypothalamo-hypophysaires. De fait, des expériences récentes démontrent que le NPY exerce, chez les amphibiens, une puissante action freinatrice sur les sécrétions du lobe intermédiaire [16] et peut donc être considéré comme un facteur hypothalamique inhibant la libération d' $\alpha$ MSH (MIF). Chez les poissons, le $\mathrm{MCH}$ inhibe également la libération d' $\alpha$-MSH $[17,18]$. L'ANF stimule in vitro la sécrétion d' $\alpha$ MSH par le lobe intermédiaire des amphibiens [19] et celle de gonadotropine par les cellules antéhypophysaires de rat [20]. Comme les fibres à $\mathrm{MCH}$ et ANF apparaissent confinées dans le lobe nerveux, il n'est pas établi que les deux neuropeptides exercent un rôle physiologique dans le contrôle des cellules mélanotropes. Cependant, le lobe intermédiaire de la grenouille, bien que très faiblement vascularisé, possède un réseau de capillaires courts qui permettent des communications humorales avec le lobe nerveux. On peut donc envisager que les neuropeptides, tels que $\mathrm{MCH}$ et $\mathrm{ANF}$, libérés par les terminaisons du lobe nerveux, diffusent jusqu'au lobe intermédiaire et agissent localement sur la sécrétion d' $\alpha$-MSH. L'ANF localisé au niveau du lobe nerveux pourrait en outre exercer un contrôle présynaptique sur la libération de vasopressine. Ainsi des études récentes montrent que, chez le rat, l'ANF inhibe la libération de vasopressine in vitro [21-23]. Enfin, des études pharmacologiques suggèrent que le NPY et l'ANF ont des activités psychotropes lorsqu'ils sont administrés par voie intracérébroventriculaire [24, 25]. Comme par ailleurs le NPY, l'ANF et le $\mathrm{MCH}$ exercent des activités biologiques variées dans divers organes périphériques [26-28], il apparaît que ces trois neuropeptides peuvent être considérés à la fois comme des hormones, des neurohormones et des neurotransmetteurs

mo 5 o vol 3, mai 87

\section{RÉFÉRENCES}

1. Prasada-Rao PD, Hartwig HG. Monoaminergic tracts of the diencephalon and innervation of the pars intermedia of the amphibian hypophysis. Cell Tissue Res 1974 ; 151 : 1-26.

2. Enemar A, Falck B. On the presence of adrenergic nerve in the pars intermedia of the frog Rana temporia. Gen Comp Endocrinol 1965 ; $5: 577-83$.

3. OErtel WH, Mugnaini E, Tappaz ML, et al. Central GABAergic innervation of neurointermediate lobe : Biochemical and immunocytochemical study in the rat. Proc Natl Acad Sci USA 1982 ; 79 : 675-9.

4. Saland LC, Wallace JC, Comunas F. Serotonin-immunoreactive nerve fibers of the rat pituitary : Effects of anticatecholamine and antiserotonin drugs on staining patterns. Brain Res $1986 ; 368: 310-8$.

5. Dierickx K, Vandesande F. Immunoenzyme cytochemical demonstration of mesotocinergic nerve fibers in the pars intermedia of the amphibian hypophysis. Cell Tissue Res 1976 ; $174: 25-33$.

6. Tonon MC, Burlet A, Lauber M, et al. Immunohistochemical localization and radioimmunoassay of corticotropin releasing factor in the forebrain and hypophysis of the frog Rana ridibundo. Neuroendocrinology 1985 ; 40 : 109-19.

7. Seki T, Nakai N, Shioda S, Mitsuma T, Kikuyama S. Distribution of immunoreactive thyrotropin-releasing hormone in the forebrain and hypophysis of the bullfrog Rana catesbeiana. Cell Tissue Res 1983; 233 : 507-16.

8. Allen YS, Adrian TE, Allen JM, et al. Neuropeptide Y : Distribution in the rat brain. Science 1983 ; 221 : 877-9.

9. Skofitsch G, Jacobowitz DM, Eskay RL Zamir N. Distribution of atrial natriuretic factor-like immunoreactive neurons in the rat brain. Neuroscience $1985 ; 16$ : 917-48.

10. Zamir N, Skofitsch G, Bannon MJ, Jacobowitz DM. Melanin-concentrating hormone : Unique peptide neuronal system in the rat brain and pituitary gland. Proc Natl Acad $S c i$ USA $1986 ; 83$ : 1528-31.

11. Danger JM, Guy J, Benyamina M, et al. Localisation and identification of neuropeptide $Y$ (NPY)-like immunoreactivity in the frog brain. Peptides 1985 ; 6 : 1225-36.

12. Netchitaïlo P, Feuilloley M, Pelletier G, et al. Atrial natriuretic factor-like immunoreactivity in the central nervous system of the frog. Neuroscience 1987 (sous presse).

13. Andersen AC, Pelletier G, Eberlé AN, Leroux $P$, Jégou S, Vaudry $H$. Localization of melanin-concentrating hormone like immunoreactivity in the brain and pituitary of the frog Rana ridibunda. Peptides $1986 ; 7$ : 941-51.

14. Danger JM, Benyamina M, Guy J, et al. Localisation et identification du neuropeptide tyrosine (NPY) dans le cerveau d'un amphibien anoure. Ann Endocrinol (Paris) 1986 ; 47 : 59-61.
15. Andersen AC, Jégou $S$, Eberlé $A N$, Tonon MC, Pelletier G, Vaudry H. Coexistence of melanin-concentrating hormone $(\mathrm{MCH})$ and alpha-melanocyte-stimulating hormone $(\alpha-\mathrm{MSH})$ in the preoptic nucleus of the frog brain. Brain Res Bull 1987; 18 (sous presse).

16. Danger JM, Leboulenger F, Guy J, et al. Neuropeptide $\mathrm{Y}$ in the intermediate lobe of the frog pituitary acts as an $\alpha$-MSH-release inhibiting factor. Life Sci 1986 ; 39 : 1183-92.

17. Baker BI, Bird DJ, Buckingham JC. Effects of chronic administration of melaninconcentrating hormone on corticotropin, melanotropin and pigmentation in the trout. Gen Comp Endocrinol 1986; 63 : 62-9.

18. Barber LD, Baker BJ, Penny JC, Eberlé AN. Melaninconcentrating hormone inhibits the release of $\alpha-\mathrm{MSH}$ from teleost pituitary glands. Gen Comp Endocrinol 1987 ; 65 : 79-86.

19. Lamacz M, Netchitaīlo $P$, Tonon MC, Feuilloley M, Cantin M, Pelletier G, Vaudry H. Atrial natriuretic factor (ANF) stimulates the release of $\alpha-\mathrm{MSH}$ from frog neurointermediate lobes in vitro. Interaction with dopamine, GABA and neuropeptide Y. Life Sci 1987 ; in press.

20. Howarth J, Ertl T, Schally AV. Effect of atrial natriuretic peptide on gonadotropin release in superfused rat pituitary cells. Proc Natl Acad Sci USA 1986 ; 83 : 3444-6.

21. Obana K, Naruse M, Inagami $T$, el al. Atrial natriuretic factor inhibits vasopressin secretion from rat posterior pituitary. Biochem Biophys Res Commun 1985; 132 : 1088-94.

22. Januszewicz P, Thibault G, Garcia R, Gutkowska J, Genest J, Cantin M. Effect of synthetic atrial natriuretic factor on vasopressin release by the rat hypothalamoneurohypophyseal complex in organ culture. Biochem Biophys Res Commun 1986 ; 134 : 652-8.

23. Crandall ME, Gregg CM. In vitro evidence for an inhibitory effect of atrial natriuretic peptide on vasopressin release. Neuroendocrinology 1986 ; 44 : 439-45.

24. Stanley BG, Leibowitz SF. Neuropeptide $Y$ injected in the paraventricular hypothalamus : A powerful stimulant of feeding behavior. Proc Natl Acad Sci USA 1985; 82 : 3940-3.

25. Antunes-Rodrigues J, McCann SM, Rogers LC, Samson WK. Atrial natriuretic factor inhibits dehydration-and angiotensin IIinduced water intake in the conscious, unrestrained rat. Proc Soc Acad Sci USA $1985 ; 82$ : 8720-3.

26. Lunberg JM, Torssell L, Sollevi A, Pernow J, Theodorsson E, Norheim E, Anggard A, Hamberger B. Neuropeptide Y and sympathetic vascular control in man. Regul Pept $1985 ; 13: 41-52$.

27. Seymour AA, Blaine EH, Mazack EK, et al. Renal and systemic effects of synthetic atrial natriuretic factor. Life Sci $1985 ; 36: 33-44$.

28. Kawauchi H, Kawazoe I, Tsubokawa M, Kishida M, Baker BI. Characterization of melanin-concentrating hormone in chum salmon pituitaries. Nature 1983 ; 305 : 321-33. 